\title{
Tendência temporal dos indicadores de excesso de peso em adultos nas capitais brasileiras, 2006-2013
}

\author{
Time trend in adult obesity indicators \\ in Brazilian state capitals, 2006-2013
}

\author{
Deborah Carvalho Malta ${ }^{1}$ \\ Maria Aline Siqueira Santos ${ }^{2}$ \\ Silvania Suely Caribé de Araújo Andrade ${ }^{2}$ \\ Taís Porto Oliveira ${ }^{2}$ \\ Sheila Rizzato Stopa ${ }^{3}$ \\ Max Moura de Oliveira ${ }^{3}$ \\ Patrícia Jaime ${ }^{3}$
}

${ }^{1}$ Departamento Materno

\begin{abstract}
The scope of this article is to analyze time trends in excess weight (overweight, obesity and class III obesity) among adults ( $\geq 18$ years of age) in Brazilian capitals between 2006 and 2013. It is a study of temporal trends in excess weight indicators using data from the telephone-based Surveillance System of Risk and Protective Factors for Chronic Non-Communicable Diseases (Vigitel). The Prais regression model was performed. In 2013, the following statistics were observed in the adult population: overweight in $32.2 \%$; obesity in 17.5\%, and class III obesity in 1.5\%. From 2006 to 2013, there was a significant increase in major indicators, for sex, age group, level of schooling (years) and regions. Overweight and obesity indicators demand attention since they result in a burden on the individual, society and health services. Key words Overweight, Obesity, Epidemiological surveys, Chronic disease
\end{abstract}

\footnotetext{
Infantil e Saúde Pública,

Escola de Enfermagem,

UFMG. R Alfredo Balena,

190, Belo Horizonte, MG.

dcmalta@uol.com.br

${ }^{2}$ Departamento de

Vigilância de Doenças e

Agravos Não Transmissíveis

e Promoção da Saúde,

Secretaria de Vigilância em

Saúde, Ministério da Saúde.

${ }^{3}$ Faculdade de Saúde

Pública, Universidade de

São Paulo. São Paulo SP

Brasil.
}

Resumo O objetivo deste artigo é analisar as tendências temporais dos indicadores de excesso de peso (sobrepeso, obesidade e obesidade grau III) entre adultos ( $\geq 18$ anos) das capitais brasileiras e do DF entre os anos de 2006 e 2013. Estudo de série temporal dos indicadores de excesso de peso, utilizando dados do inquérito telefônico para Vigilância de Fatores de Risco e Proteção para Doenças Crônicas (Vigitel). Foi realizado modelo de regressão de Prais. Em 2013, observou-se na população adulta: sobrepeso em 32,2\%, obesidade em 17,5\% e obesidade grau III em 1,5\%. De 2006 a 2013, ocorreu aumento estatisticamente significante na maioria dos indicadores, para ambos os sexos, faixas etárias, escolaridade e regiões. Os indicadores de excesso de peso, obesidade requerem atenção, pois acarretam ônus ao indivíduo, à sociedade e aos serviços de saúde.

Palavras-chave Sobrepeso, Obesidade, Inquéritos epidemiológicos, Doença crônica 


\section{Introdução}

No mundo, em 2012, 38 milhões de pessoas faleceram em razão das Doenças Crônicas Não Transmissiveis (DCNT), com destaque para as enfermidades do aparelho circulatório, diabetes, câncer e doença respiratória crônica ${ }^{1}$. Um dos fatores de risco responsáveis pelo aumento da morbidade e mortalidade por DCNT é a obesidade, constituindo-se um desafio mundial controlar sua progressão $0^{2,3}$. Nas Américas, vem sendo observado um aumento da obesidade, independente do nível de desenvolvimento do país4.

No Brasil, as DCNT foram responsáveis por $72,4 \%$ dos óbitos no ano de $2011^{5}$, representando a principal fonte da carga de doença no país 6 . No período de 2002 a 2012, ocorreram 5.887 .708 internações relacionadas às DCNT em 2002 e 6.411.791 em 2012, com tendência crescente. Análises descritivas do Sistema de Informação Ambulatorial do Sistema Único de Saúde para os anos de 2008 a 2012 apontam crescimento exponencial nos atendimentos ambulatoriais relacionados às DCNT, principalmente aqueles referentes às doenças cardiovasculares e às cirurgias bariátricas ${ }^{7}$.

Para efeitos de medidas populacionais em adultos, o excesso de peso e a obesidade são mensurados pelo Índice de Massa Corporal (IMC) (definido pelo peso em quilos dividido pela altura em metros quadrados), independente do sexo e idade, e possui boa correlação com as medidas da gordura corporal ${ }^{8,9}$.

O excesso de peso é multifatorial, resultante da interação complexa entre fatores genéticos, metabólicos, hormonais, ambientais, comportamentais, culturais e sociais. Destacam-se o consumo de alimentos processados de alta densidade calórica aliada a um menor consumo de fibras e redução do gasto energético em função dos baixos níveis de atividade física ${ }^{1,10}$. Estudos apontam que os determinantes sociais são preponderantes na gênese da obesidade; como, por exemplo, baixa escolaridade, menor renda, união conjugal (casados e viúvos) e envelhecimento, dentre outros. A relação entre os determinantes sociais e a obesidade é complexa e ainda não está totalmente esclarecida. Há hipóteses de que a obesidade pode ser desencadeada como sequela da desnutrição energético-proteica precoce, ou pelo desequilíbrio no gasto energético/ingesta calórica, ou relacionada a fatores genéticos ${ }^{4,8}$.

A obesidade é considerada um problema de saúde pública no Brasil e a transição nutricional se deu de forma muito rápida ${ }^{11}$. Dados da Pes- quisa de Orçamentos Familiares (POF), 20082009, já apontavam que 49,0\% da população adulta brasileira apresentavam excesso de peso e $14,8 \%$ eram obesos ${ }^{12}$. Além desses dados, o Sistema de Vigilância de Fatores de Risco e Proteção para Doenças Crônicas por Inquérito Telefônico (Vigitel) realizado nas 26 capitais brasileiras e no Distrito Federal, apresenta dados desde 2006 sobre estilo de vida em adultos e informações antropométricas (peso e altura) autorreferidas. Esse sistema possibilita o monitoramento dos indicadores de excesso de peso/obesidade nesta população e subsidia o planejamento e a avaliação de políticas públicas de promoção e prevenção da saúde na área de $\mathrm{DCNT}^{13}$.

Este estudo teve como objetivo analisar as tendências temporais dos indicadores de excesso de peso (sobrepeso, obesidade e obesidade grau III) entre adultos ( $\geq 18$ anos) das capitais brasileiras e do DF entre os anos de 2006 e 2013.

\section{Métodos}

Foi realizado um estudo de série temporal dos indicadores de excesso de peso, utilizando dados autorreferidos do Sistema de Vigilância de fatores de risco e proteção para doenças crônicas por inquérito telefônico, no período de 2006 a 2013, para a população de adultos nas 26 capitais brasileiras e no Distrito Federal.

O processo de amostragem do Vigitel é probabilístico e realizado em duas etapas: 1) sorteio de 5.000 linhas de telefone fixo residencial em cada uma das 26 capitais e no DF; 2) seleção de um adulto ( $\geq 18$ anos de idade) morador do domicílio para responder ao questionário. $\mathrm{O}$ número de entrevistas realizadas em cada uma das cidades por ano foi: 54.369 (2006), 54.251 (2007), 54.353 (2008), 54.367 (2009), 54.339 (2010), 54.144 (2011), 45.448 (2012) e 52.929 (2013). Para que os resultados obtidos fossem representativos de todos os adultos de cada cidade pesquisada, foram utilizados pesos pós-estratificação compostos pelas variáveis: sexo, faixa etária e escolaridade; de modo a igualar a distribuição sociodemográfica da amostra à distribuição estimada para a população adulta de cada cidade ${ }^{13}$.

As prevalências analisadas neste estudo foram calculadas com base no IMC. Tanto o peso quanto a altura foram informações autorreferidas. Foi realizada a imputação de dados faltantes de peso e altura a partir do ano de 2012. O método de imputação utilizado foi o hot deck, onde é identificada a associação entre as variáveis ida- 
de, sexo, escolaridade e raça/cor da pele e a não resposta para peso e/ou altura. Posteriormente, é feita a seleção aleatória dentro de cada grupo, de um indivíduo com informações conhecidas para replicar seus valores de peso ou altura para o não respondente do mesmo grupo ${ }^{13}$. No ano de 2012 houve 3.921 dados faltantes de peso e altura e em 2013, 4.661, correspondendo aos percentuais de $8,6 \%$ e $8,8 \%$, respectivamente.

Mais detalhes metodológicos podem ser vistos no relatório anual do Vigitel ${ }^{13}$. As definições utilizadas para os indicadores de excesso de peso estudados foram as seguintes ${ }^{12,13}$ :

a) Sobrepeso: indivíduo com IMC entre $25 \mathrm{e}$ $30 \mathrm{~kg} / \mathrm{m}^{2}$;

b) Obesidade (incluindo grau I, grau II e grau III): indivíduo com IMC $\geq 30 \mathrm{~kg} / \mathrm{m}^{2}$;

c) Obesidade grau III (obesidade mórbida): indivíduo com IMC $\geq 40 \mathrm{~kg} / \mathrm{m}^{2}$.

Para análise de tendência dos indicadores de excesso de peso, foi utilizada modelagem de regressão de Prais-Winsten, com o objetivo de corrigir o efeito da autocorrelação. As tendências foram classificadas como ascendente ( $p \leq 0,05$ e coeficiente de regressão positivo), em declínio
( $\mathrm{p} \leq 0,05$ e coeficiente de regressão negativo) e estável ( $p>0,05)$. Foram calculadas as variações percentuais médias anuais das prevalências empregando-se os valores obtidos na regressão ${ }^{14}$.

Para as análises estatísticas utilizou-se o aplicativo "Stata” versão 12.1. O Vigitel foi aprovado pela Comissão Nacional de Ética em Pesquisa em Seres Humanos.

\section{Resultados}

As Tabelas de 1 a 3 apresentam as prevalências encontradas para os indicadores de excesso de peso estudados na população adulta residente nas 26 capitais brasileiras e no Distrito Federal. Segundo esses dados, a prevalência de sobrepeso foi de 30,9\% e 33,2\%, em 2006 e 2013, respectivamente, com elevação de 7,4\% (Tabela 1). Com relação à obesidade, o aumento foi de 47,1\% no mesmo período, alcançando $17,5 \%$ da população em 2013 (Tabela 2). Para a obesidade grau III os valores observados em 2006 e 2013 foram, respectivamente, $1,1 \%$ e $1,5 \%$, com $36,4 \%$ de crescimento (Tabela 3 ).

Tabela 1. Tendência da Prevalência (\%) de sobrepeso (Índice de Massa Corporal $\geq 25 \mathrm{e}<30 \mathrm{~kg} / \mathrm{m}^{2}$ ) no conjunto da população adulta ( $\geq 18$ anos) residente nas capitais brasileiras e Distrito Federal, Vigitel, 2006-2013.

\begin{tabular}{|c|c|c|c|c|c|c|c|c|}
\hline \multirow{2}{*}{ Variáveis } & \multicolumn{8}{|c|}{ Anos } \\
\hline & 2006 & 2007 & 2008 & 2009 & 2010 & 2011 & 2012 & 2013 \\
\hline \multicolumn{9}{|l|}{ Sexo } \\
\hline Masculino & 36,2 & 35,1 & 36,4 & 36,3 & 38,0 & 37,9 & 38,0 & 37,2 \\
\hline Feminino & 26,3 & 25,7 & 26,8 & 27,7 & 29,0 & 28,4 & 29,9 & 29,8 \\
\hline Ambos & 30,9 & 30,0 & 31,2 & 31,6 & 33,1 & 32,8 & 33,6 & 33,2 \\
\hline \multicolumn{9}{|l|}{ Faixa etária } \\
\hline 18 a 24 & 16,4 & 16,8 & 18,3 & 19,0 & 22,0 & 20,0 & 21,3 & 23,4 \\
\hline 25 a 34 & 27,7 & 28,4 & 29,8 & 29,5 & 32,1 & 32,3 & 32,6 & 30,2 \\
\hline 35 a 44 & 35,8 & 33,1 & 34,2 & 34,8 & 35,2 & 35,4 & 36,2 & 36,4 \\
\hline 45 a 54 & 38,5 & 35,7 & 36,7 & 37,3 & 36,3 & 36,5 & 38,3 & 38,2 \\
\hline 55 a 64 & 39,2 & 37,1 & 37,9 & 37,8 & 40,6 & 39,2 & 36,8 & 38,3 \\
\hline 65 e mais & 35,6 & 35,9 & 36,2 & 36,5 & 37,2 & 36,6 & 39,5 & 36,0 \\
\hline \multicolumn{9}{|l|}{ Escolaridade } \\
\hline 0 a 8 & 33,7 & 32,7 & 32,8 & 34,0 & 35,4 & 34,7 & 35,6 & 35,8 \\
\hline 9 a 11 & 28,4 & 26,4 & 29,7 & 30,0 & 31,4 & 31,6 & 31,5 & 32,2 \\
\hline 12 e mais & 28,6 & 30,3 & 30,5 & 29,9 & 31,8 & 31,6 & 34,0 & 31,2 \\
\hline \multicolumn{9}{|l|}{ Região } \\
\hline Norte & 28,6 & 29,1 & 30,5 & 31,0 & 31,8 & 32,7 & 33,1 & 34,3 \\
\hline Nordeste & 28,6 & 29,3 & 29,9 & 30,7 & 31,5 & 32,1 & 33,1 & 32,8 \\
\hline Centro-Oeste & 29,9 & 29,5 & 30,5 & 30,7 & 33,7 & 33,8 & 33,6 & 33,3 \\
\hline Sudeste & 32,4 & 30,5 & 32,0 & 32,3 & 34,1 & 32,5 & 33,7 & 32,9 \\
\hline Sul & 33,0 & 31,1 & 32,6 & 33,3 & 33,7 & 34,7 & 35,2 & 35,3 \\
\hline
\end{tabular}


Tabela 2. Tendência da Prevalência (\%) de obesidade (Índice de Massa Corporal $\geq 30 \mathrm{~kg} / \mathrm{m}^{2}$ ) no conjunto da população adulta ( $\geq 18$ anos) residente nas capitais brasileiras e Distrito Federal, Vigitel, 2006-2013.

\begin{tabular}{|c|c|c|c|c|c|c|c|c|}
\hline \multirow{2}{*}{ Variáveis } & \multicolumn{8}{|c|}{ Anos } \\
\hline & 2006 & 2007 & 2008 & 2009 & 2010 & 2011 & 2012 & 2013 \\
\hline \multicolumn{9}{|l|}{ Sexo } \\
\hline Masculino & 11,4 & 13,6 & 13,4 & 13,9 & 14,4 & 15,5 & 16,5 & 17,5 \\
\hline Feminino & 12,2 & 13 & 13,9 & 14,7 & 15,6 & 16,5 & 18,2 & 17,5 \\
\hline Ambos & 11,9 & 13,3 & 13,7 & 14,3 & 15,1 & 16 & 17,4 & 17,5 \\
\hline \multicolumn{9}{|l|}{ Faixa etária } \\
\hline 18 a 24 & 4,3 & 4,2 & 4,8 & 6,6 & 5,7 & 5,7 & 7,5 & 6,3 \\
\hline 25 a 34 & 9,9 & 11,3 & 11,2 & 11,9 & 12,2 & 13,7 & 15,1 & 15 \\
\hline 35 a 44 & 12,7 & 15,1 & 15,2 & 15,6 & 16,6 & 19,6 & 19,7 & 20,1 \\
\hline 45 a 54 & 16,2 & 19,4 & 18,6 & 17,9 & 21,6 & 21,2 & 22,6 & 22,5 \\
\hline 55 a 64 & 17,6 & 19,9 & 20,8 & 21,6 & 19,8 & 21,1 & 23,4 & 24,4 \\
\hline 65 e mais & 16,8 & 14,9 & 17,4 & 17,7 & 19,4 & 17,7 & 19 & 20,2 \\
\hline \multicolumn{9}{|l|}{ Escolaridade } \\
\hline 0 a 8 & 15,3 & 16,9 & 17,5 & 18,1 & 18,8 & 19,7 & 21,7 & 22,3 \\
\hline 9 a 11 & 9,1 & 10,7 & 10,9 & 12,2 & 13,1 & 14,2 & 15,2 & 15,1 \\
\hline 12 e mais & 8,7 & 9,9 & 10,2 & 10,7 & 11,7 & 13 & 14,4 & 14,3 \\
\hline \multicolumn{9}{|l|}{ Região } \\
\hline Norte & 13,3 & 14,1 & 14,4 & 14,9 & 16,5 & 17 & 18 & 17,6 \\
\hline Nordeste & 12 & 12,9 & 13,6 & 14,3 & 15,4 & 16 & 17,1 & 16,5 \\
\hline Centro-Oeste & 11 & 12,1 & 12,6 & 11,5 & 12,8 & 15,1 & 15,7 & 16,5 \\
\hline Sudeste & 11,6 & 13,7 & 13,7 & 15,1 & 14,9 & 15,9 & 17,9 & 18,3 \\
\hline Sul & 12,4 & 13,2 & 14,2 & 13,8 & 16,1 & 16,8 & 17,1 & 17,4 \\
\hline
\end{tabular}

Tabela 3. Tendência da Prevalência (\%) de obesidade grau III (Índice de Massa Corporal $\geq 40 \mathrm{~kg} / \mathrm{m}^{2}$ ) no conjunto da população adulta ( $\geq 18$ anos) residente nas capitais brasileiras e Distrito Federal, Vigitel, 2006-2013.

\begin{tabular}{|c|c|c|c|c|c|c|c|c|}
\hline \multirow{2}{*}{ Variáveis } & \multicolumn{8}{|c|}{ Anos } \\
\hline & 2006 & 2007 & 2008 & 2009 & 2010 & 2011 & 2012 & 2013 \\
\hline \multicolumn{9}{|l|}{ Sexo } \\
\hline Masculino & 0,9 & 0,8 & 0,5 & 0,7 & 0,8 & 0,8 & 1,0 & 1,2 \\
\hline Feminino & 1,3 & 1,2 & 1,3 & 1,6 & 1,6 & 1,4 & 2,0 & 1,8 \\
\hline Ambos & 1,1 & 1,0 & 0,9 & 1,2 & 1,2 & 1,2 & 1,5 & 1,5 \\
\hline \multicolumn{9}{|l|}{ Faixa etária } \\
\hline 18 a 24 & 0,3 & 0,5 & 0,6 & 0,5 & 0,3 & 0,3 & 0,3 & 0,3 \\
\hline 25 a 34 & 0,9 & 0,9 & 0,7 & 1,0 & 0,9 & 1,0 & 1,4 & 1,1 \\
\hline 35 a 44 & 0,9 & 1,0 & 1,1 & 1,2 & 1,3 & 1,2 & 1,6 & 1,8 \\
\hline 45 a 54 & 1,7 & 1,7 & 0,9 & 1,7 & 1,9 & 1,9 & 1,8 & 2,1 \\
\hline 55 a 64 & 1,6 & 1,2 & 1,6 & 1,4 & 1,9 & 1,2 & 2,7 & 2,4 \\
\hline 65 e mais & 1,9 & 1,0 & 1,1 & 1,6 & 1,6 & 1,3 & 1,8 & 1,9 \\
\hline \multicolumn{9}{|l|}{ Escolaridade } \\
\hline 0 a 8 & 1,8 & 1,6 & 1,2 & 1,8 & 2,0 & 1,5 & 2,1 & 2,4 \\
\hline 9 a 11 & 0,5 & 0,5 & 0,8 & 0,9 & 0,7 & 0,9 & 1,4 & 0,9 \\
\hline 12 e mais & 0,5 & 0,6 & 0,6 & 0,6 & 0,7 & 0,9 & 0,9 & 1,2 \\
\hline \multicolumn{9}{|l|}{ Região } \\
\hline Norte & 1,3 & 1,1 & 1,2 & 1,4 & 1,4 & 1,3 & 1,8 & 1,3 \\
\hline Nordeste & 0,9 & 1,2 & 1,2 & 1,4 & 1,5 & 1,3 & 1,3 & 1,5 \\
\hline Centro-Oeste & 0,6 & 0,8 & 0,9 & 0,8 & 0,8 & 1,0 & 1,3 & 1,2 \\
\hline Sudeste & 1,2 & 0,8 & 0,6 & 1,0 & 1,1 & 1,1 & 1,7 & 1,7 \\
\hline Sul & 1,1 & 0,8 & 1,2 & 0,9 & 1,1 & 0,9 & 1,3 & 1,3 \\
\hline
\end{tabular}


A prevalência de sobrepeso e de obesidade aumentou significativamente em ambos os sexos e em todos os níveis de escolaridade e em todas as regiões geográficas no período estudado (Tabela 4). Nos indivíduos das faixas etárias de 18 a 24 anos e de 25 a 34 anos houve uma tendência

Tabela 4. Tendência das prevalências dos indicadores de excesso de peso segundo características selecionadas. Brasil, 2006-2013.

\begin{tabular}{|c|c|c|c|}
\hline \multirow{2}{*}{ Variáveis } & \multicolumn{3}{|c|}{ Sobrepeso } \\
\hline & Variação média anual (\%) & IC95\% & Interpretação \\
\hline Total & 2,34 & $0,31-7,53$ & Aumento \\
\hline \multicolumn{4}{|l|}{ Sexo } \\
\hline Masculino & 3,47 & $1,06-8,73$ & Aumento \\
\hline Feminino & 1,30 & $0,43-8,27$ & Aumento \\
\hline \multicolumn{4}{|l|}{ Faixa etária } \\
\hline 18 a 24 & 7,62 & $0,65-43,95$ & Aumento \\
\hline 25 a 34 & 2,83 & $0,77-62,72$ & Aumento \\
\hline 35 a 44 & 1,10 & $0,56-9,11$ & Aumento \\
\hline 45 a 54 & 0,36 & $-0,83-10,11$ & Estabilidade \\
\hline 55 a 64 & $-0,02$ & $-0,95-16,79$ & Estabilidade \\
\hline 65 e mais & 0,60 & $0,40-0,74$ & Aumento \\
\hline \multicolumn{4}{|c|}{ Escolaridade (anos de estudo) } \\
\hline 0 a 8 & 1,78 & $0,44-0,91$ & Aumento \\
\hline 9 a 11 & 4,52 & $0,03-0,97$ & Aumento \\
\hline 12 e mais & 2,70 & $0,36-9,03$ & Aumento \\
\hline \multicolumn{4}{|l|}{ Regiões geográficas } \\
\hline Norte & 4,27 & $1,40-10,55$ & Aumento \\
\hline Nordeste & 4,20 & $3,57-4,91$ & Aumento \\
\hline Centro-Oeste & 3,63 & $0,52-43,34$ & Aumento \\
\hline Sudeste & 1,15 & $0,46-7,55$ & Aumento \\
\hline Sul & 2,67 & $0,21-10,14$ & Aumento \\
\hline \multirow{2}{*}{ Variáveis } & \multicolumn{3}{|c|}{ Obesidade } \\
\hline & Variação média anual (\%) & IC95\% & Interpretação \\
\hline Total & 5,35 & $2,67-9,98$ & Aumento \\
\hline \multicolumn{4}{|l|}{ Sexo } \\
\hline Masculino & 7,16 & $7,01-7,31$ & Aumento \\
\hline Feminino & 4,61 & $4,16-5,06$ & Aumento \\
\hline \multicolumn{4}{|l|}{ Faixa etária } \\
\hline 18 a 24 & 1,61 & $1,15-2,07$ & Aumento \\
\hline 25 a 34 & 4,55 & $4,06-5,04$ & Aumento \\
\hline 35 a 44 & 10,48 & $9,79-11,16$ & Aumento \\
\hline 45 a 54 & 5,89 & $5,16-6,62$ & Aumento \\
\hline 55 a 64 & 5,21 & $4,02-6,40$ & Aumento \\
\hline 65 e mais & 2,80 & $2,14-3,45$ & Aumento \\
\hline \multicolumn{4}{|c|}{ Escolaridade (anos de estudo) } \\
\hline 0 a 8 & 8,12 & $7,67-8,58$ & Aumento \\
\hline 9 a 11 & 7,19 & $6,99-7,40$ & Aumento \\
\hline 12 e mais & 5,99 & $6,42-5,56$ & Aumento \\
\hline \multicolumn{4}{|l|}{ Regiões geográficas } \\
\hline Norte & 4,27 & $4,61-3,93$ & Aumento \\
\hline Nordeste & 5,21 & $5,11-5,32$ & Aumento \\
\hline Centro-Oeste & 5,02 & $4,49-5,35$ & Aumento \\
\hline Sudeste & 6,40 & $5,95-6,84$ & Aumento \\
\hline Sul & 5,03 & $4,61-5,45$ & Aumento \\
\hline
\end{tabular}




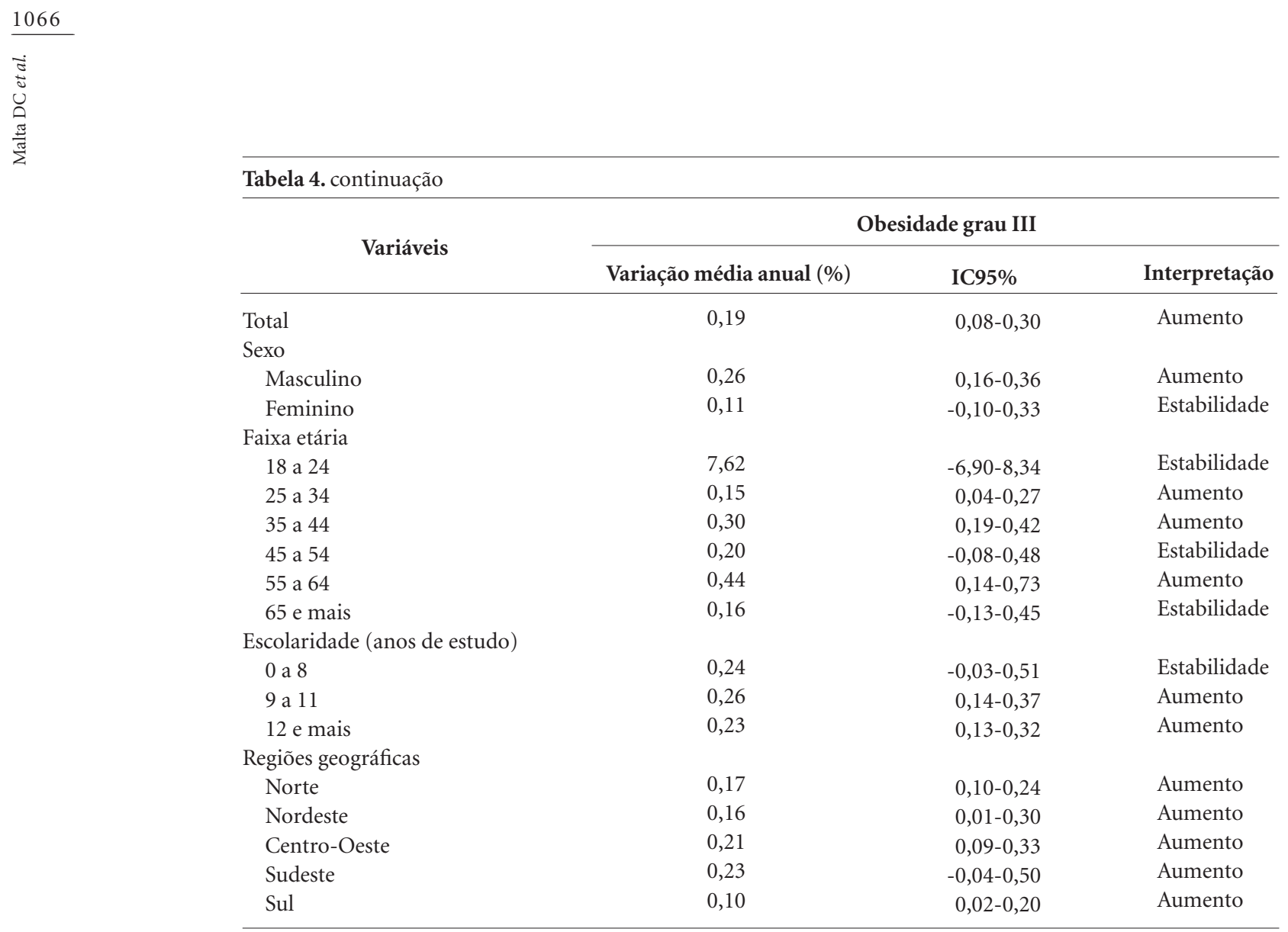

crescente na prevalência de sobrepeso. Todavia, a prevalência de obesidade aumenta significativamente em todas as faixas etárias. Apenas para o conjunto de capitais da região Sudeste houve tendência de estabilidade na prevalência de sobrepeso (Tabela 4). Todas as regiões mostraram aumento na tendência de obesidade ao longo do período avaliado (Tabela 2).

Ocorreu incremento significativo na prevalência de obesidade grau III entre mulheres, na faixa etária de 35 a 44 anos, e entre os adultos de maior escolaridade (acima de nove anos de estudo) e nas regiões Sudeste, Centro-Oeste e Nordeste (Tabela 4).

Para a população adulta das capitais, a tendência do excesso de peso foi crescente no período estudado de uma forma geral. Considerando o conjunto de capitais e Distrito Federal, houve crescimento na prevalência de todos os indicadores de excesso de peso entre os adultos pesquisados, independente do sexo, exceto no indicador de obesidade grau III, no qual o sexo masculino teve comportamento estável ao longo do período. No entanto, salienta-se que os indicadores excesso de peso mantiveram-se estáveis entre os anos de 2012 e 2013 (Tabela 4).

\section{Discussão}

Os dados do estudo apontam que em 2013, um terço da população adulta apresentou sobrepeso, cerca de um quinto obesidade e 1,5\% obesidade grau III. Ocorreu aumento estatisticamente significativo na maioria dos indicadores, para ambos os sexos, faixas etárias, escolaridade e regiões no período entre 2006 e 2013.

A pandemia da obesidade é um fenômeno global, sendo verificada por meio de altas prevalências de obesidade na população adulta em países como os Estados Unidos (35\%); México (34,5\% em homens e 24,2\% em mulheres); Canadá (27,6\% em homens e $23,5 \%$ em mulheres); Austrália (25,6\% em homens e 24\% em mulheres), Espanha $(24,4 \%$ em homens e $32,5 \%$ em mulheres) e Paraguai (22,9\% em homens e $35,7 \%$ em mulheres); dentre outros ${ }^{15}$.

Quando se analisa todo o período, de 2006 a 2013, houve tendência de crescimento tanto para o sobrepeso quanto para a obesidade em ambos os sexos. Dados de países europeus, baseados em peso medido e referido, apontam maiores prevalências de obesidade e sobrepeso entre mulheres $^{15}$. No presente estudo, a prevalência da obesi- 
dade foi semelhante entre homens e mulheres e o sobrepeso foi maior em homens.

Observou-se associação inversa entre escolaridade (proxy de nível socioeconômico) e os indicadores de excesso de peso, como também encontrado no Canadá ${ }^{16,17}$. Este achado tem sido explicado pelo poder aquisitivo inferior das populações com baixa escolaridade, que acaba por consumir alimentos de baixo custo e altamente energéticos, ao invés de consumir outros mais saudáveis, como frutas, hortaliças e cereais integrais. Além disto, há menos oportunidade para a prática de atividade física no lazer, menor acesso a informações sobre medidas preventivas, dentre outros fatores ${ }^{17}$. Destaca-se ainda que nos países de baixa e média rendas, a obesidade acentua as desigualdades em saúde, levando a maior carga de morbimortalidade em populações de baixo nível socioeconômico ${ }^{18}$. A relação inversa entre a prevalência de excesso de peso e obesidade e o nível de escolaridade verificada no Vigitel para as mulheres, foi descrita anteriormente em estudos nacionais ${ }^{16,19}$.

A prevalência de sobrepeso e obesidade no Brasil aumentou com a idade. Este padrão tem sido descrito em outros estudos ${ }^{20,21}$. A relação entre ingesta de alimentos e gasto energético é uma das explicações prováveis para a relação direta entre o aumento do sobrepeso e da obesidade. Com o envelhecimento, o nível de atividade física se reduz. Fatores relacionados a este padrão citados pela literatura são: crescimento do consumo de alimentos fora de casa; aumento na oferta de refeições rápidas, com elevado teor energético; redução da atividade física no lazer, meios de deslocamento motorizados; predomínio crescente das ocupações que demandam menor esforço físico, dentre outros ${ }^{20,21}$.

Quanto à obesidade grau III, no ano de 2013 a prevalência foi de 1,5\%, ocorrendo um aumento significativo no período. Estudo realizado a partir de comparação de três bases populacionais aponta um crescimento de $255 \%$ na prevalência de obesidade grau III nos últimos 30 anos, com as maiores prevalências encontradas na região Sudeste $^{22}$, o que corrobora com os dados deste estudo.

Dentre os inúmeros compromissos globais com o tema de DCNT e obesidade, destaca-se o plano global de enfrentamento de DCNT da Organização Mundial da Saúde (OMS), que tem como meta deter o excesso de peso e a obesidade entre as pessoas com 18 anos ou mais e entre os adolescentes ${ }^{2}$. No Brasil, em 2011, foi lançado o Plano de Ações Estratégicas para o Enfrenta- mento das Doenças Crônicas não Transmissíveis (DCNT) 2011-2022 ${ }^{18}$, e em 2013 as diretrizes descritas no Plano Intersetorial para Prevenção e Controle da Obesidade? .

O Plano de Enfrentamento de DCNT no Brasil definiu como meta deter o crescimento da obesidade em adultos e de redução do excesso de peso e obesidade em adolescentes e crianças, conforme diretriz da $\mathrm{OMS}^{23}$. Em consonância com os Planos citados, em 2013, o Ministério da Saúde publicou portaria que trata da organização da prevenção e do tratamento das pessoas com sobrepeso e da obesidade como linha de cuidado prioritária da rede de Atenção à Saúde das Pessoas com Doenças Crônicas9.

Outra proposta para ampliar o comprometimento com programas e ações nesse campo, é a atuação junto às famílias, escolas e profissionais de saúde, no incentivo ao aleitamento materno, na regulamentação da propaganda de alimentos com alto teor de gordura, no estímulo à prática de atividades físicas, e na promoção da alimentação adequada e saudável em todos os ciclos da vida $^{20}$. Em conformidade e seguindo a recomendação da OMS de formular e atualizar diretrizes nacionais sobre alimentação e nutrição ${ }^{24}$, foi realizada a revisão do Guia Alimentar para a População Brasileira de 2006 e publicada uma nova edição em $2014^{25}$.

O uso de medidas autorreferidas pode ser visto como uma limitação deste estudo. Entretanto, diversas pesquisas atestam a validade dessa estratégia ${ }^{11}$. Outras limitações são inerentes ao método utilizado pelo Vigitel, que entrevista indivíduos residentes nas capitais de estados brasileiros e no Distrito Federal que possuem telefone fixo. No entanto, para ampliar a representatividade dessa amostra, são utilizados uso de fatores de ponderação, com o objetivo de igualar as características demográficas da amostra àquelas da população de adultos de cada cidade estudada, segundo dados do censo IBGE 2010. Pode ter ocorrido um viés, pois o IMC dos indivíduos que possuem telefone fixo pode ser diferente daqueles que não o possuem ${ }^{13}$.

\section{Conclusão}

O incremento no sobrepeso e na obesidade requer atenção, pois acarreta ônus ao indivíduo, à sociedade e aos serviços de saúde. Há a necessidade de incentivos à adoção de práticas de promoção de saúde e estilo de vida saudável, já que estas refletem de maneira positiva na qualidade 
de vida da população. Ainda, é importante salientar que considerações a respeito de políticas e ações direcionadas à população, como a regulamentação que estimula a redução da quantidade de gordura trans e açúcar nos alimentos, são necessárias para melhorar as condições de vida e saúde desta população.

\section{Colaboradores}

DC Malta, MAS Santos, SSCA Andrade, TP Oliveira, SR Stopa, MM Oliveira e P Jaime participaram igualmente da elaboração e análise dos dados descritos neste manuscrito. 


\section{Referências}

1. World Health Organization (WHO). Noncommunicable diseases country profiles 2014. Geneva: WHO; 2014. [acessado 2015 ago 31].Disponível em: http://apps.who. int/iris/bitstream/10665/128038/1/9789241507509_ eng.pdf?ua $=1$

2. World Health Organization (WHO). Global status report on noncommunicable diseases 2010. Geneva: WHO; 2011.

3. World Health Organization (WHO). Prevention and control of non communicable diseases: Formal meeting of Member States to conclude the work on the comprehensive global monitoring framework, including indicators, and a set of voluntary global targets for the prevention and control of non communicable diseases. Geneva: WHO; 2012. [acessado 2013 jan 18]. Disponível em: http://apps.who.int/gb/NCDs/pdf/A_NCD_2-en.pdf.

4. Pinheiro ARO, Freitas SFT, Corso ACT. Uma abordagem epidemiológica da obesidade. Rev Nutr 2004; 17(4):523-533.

5. Malta DC, Moura L, Prado RR, Escalante JC, Schmidt MI, Duncan BB. Mortalidade por doenças crônicas não transmissíveis no Brasil e suas regiões, 2000 a 2011. Epidemiol Serv Saúde 2014; 23(4):599-608.

6. Schmidt MI, Duncan BB, Silva GA, Menezes AM, Monteiro CA, Barreto SM, Chor D, Menezes PR. Doenças crônicas não transmissíveis no Brasil: carga e desafios atuais. Lancet 2011; 61-74.

7. Malta DC, Prestes IV, Oliveira JCG, Moura L, Nunes ML, Oliveira MM, Andrade SSCA, Anjos TG. Morbidade Hospitalar e Ambulatorial em Doenças Crônicas não Transmissíveis no Sistema Único de Saúde- DCNT. In: Ministério da Saúde (MS). Saúde Brasil 2012. Brasília: MS; 2013. p. 243-271.

8. World Health Organization (WHO). Obesity: preventing and managing the global epidemic. Report a WHO Consultation on Obesity. Geneva: WHO; 2000.

9. Brasil. Ministério da Saúde. Gabinete do Ministro. Portaria no 424, de 19 de março de 2013. Redefine as diretrizes para a organização da prevenção e do tratamento do sobrepeso e obesidade como linha de cuidado prioritária da Rede de Atenção à Saúde das Pessoas com Doenças Crônicas. Diário Oficial da União 2013; 20 mar.

10. Barbosa LS, Scala LCN, Ferreira MC. Associação entre marcadores antropométricos de adiposidade corporal e hipertensão arterial na população adulta de Cuiabá, Mato Grosso. Rev Bras Epidemiol 2009; 12(2):237-247.

11. Batista Filho M, Rissin A. A transição nutricional no Brasil: tendências regionais e temporais. Cad Saude Publica 2003; 19(Supl. 1):S181-191.

12. Gigante DP, França GVA, Sardinha LMV, Iser BPM, Meléndez GV. Variação temporal na prevalência do excesso de peso e obesidade em adultos: Brasil, 2006 a 2009. Rev Bras Epidemiol 2011; 14(Supl. 1):157-165.

13. Brasil. Ministério da Saúde (MS). Secretaria de Vigilância em Saúde. Departamento de Vigilância de Doenças e Agravos Não Transmissíveis e Promoção da Saúde. Vigitel Brasil 2013: Vigilância de Fatores de Risco e Proteção para Doenças Crônicas por Inquérito Telefônico. Brasília: MS; 2014.
14. Antunes JLF, Waldman EA. Trends and spatial distribution of deaths of children aged 12-60 months in São Paulo, Brazil, 1980-98. Bull World Health Organ 2002; 80(5):391-398.

15. International Association for the Study of Obesity. Adult overweight and obesity in the European Union (EU27). IASO; 2013. [acessado 2014 nov 10]. Disponível em: http://www.worldobesity.org/site_media/library/resource_images/EU_27_Adults_Dec_2014.pdf

16. Instituto Brasileiro de Geografia e Estatística (IBGE). Pesquisa de Orçamentos Familiares 2008-2009: antropometria e estado nutricional de crianças, adolescentes e adultos no Brasil. Rio de Janeiro: IBGE; 2010.

17. Tjepkema M. Measured Obesity. Adult obesity in Canada: measured height and weight. Statistics Canada Catalogue. 2007. [acessado 2014 nov 10]. Disponível em: http://www.aboutmen.ca/application/www.aboutmen. ca/asset/upload/tiny_mce/page/link/Adult-Obesity-inCanada.pdf

18. Brasil. Ministério da Saúde (MS). Secretaria de Vigilância em Saúde. Departamento de Análise de Situação de Saúde. Plano de ações estratégicas para o enfrentamento das doenças crônicas não transmissíveis (DCNT) no Brasil, 2011-2022. Brasília: MS; 2011.

19. Gigante DP, Moura EC, Sardinha LMV. Prevalência de excesso de peso e obesidade e fatores associados, Brasil, 2006. Rev Saude Publica 2009; 43(Supl. 2):83-89.

20. Malta DC, Silva Júnior JB. Policies to promote physical activity in Brazil. Lancet 2012; 380(9838):195-196.

21. Jaime PC, da Silva ACF, Gentil PC, Claro RM, Monteiro AC. Brazilian obesity prevention and control initiatives obesity reviews. Obes Rev 2013; 14(Supl. 2):88-95.

22. Santos LMP, Oliveira IV, Peters LR, Conde WL. Trends in Morbid Obesity and in Bariatric Surgeries Covered by the Brazilian Public Health System. Obes Surg 2010; 20(7):943-948.

23. Malta DC, Silva Júnior JB. O Plano de Ações Estratégicas para o Enfrentamento das Doenças Crônicas Não Transmissíveis no Brasil e a definição das metas globais para o enfrentamento dessas doenças até 2025: uma revisão. Epidemiol Serv Saude 2013; 22(1):151-164.

24. World Health Organization (WHO). Global Strategy on diet, physical activity and health. Geneva: WHO; 2004.

25. Brasil. Ministério da Saúde (MS). Secretaria de Atenção à Saúde. Departamento de Atenção Básica. Guia Alimentar para a População Brasileira. 2a ed. Brasília: MS; 2014.

Artigo apresentado em 16/06/2015

Aprovado em 08/09/2015

Versão final apresentada em 10/09/2015 
